\title{
Iron deficiency and cognition
}

\author{
Lena Hulthén
}

Department of Clinical Nutrition, Sahlgrenska Academy at Göteborg University, Göteborg, Sweden

\section{Abstract}

Iron deficiency is the most prevalent nutritional disorder in the world. One of the most worrying consequences of iron deficiency in children is the alteration of behaviour and cognitive performance. In iron-deficient children, striking behavioural changes are observed, such as reduced attention span, reduced emotional responsiveness and low scores on tests of intelligence. Animal studies on nutritional iron deficiency show effects on learning ability that parallel the human studies. Despite the obvious confounding impact of socioeconomic factors, there is a wealth of clinical, biochemical and neuropathological research that shows that iron deficiency can exert a direct deleterious effect on learning and the brain, and that this can occur with normal haemoglobin levels. Nutritional iron replacement therapy readily corrects haematological iron status in iron-deficient children within 2-3 weeks, but behavioural problems persist for several months or years. This resistance to iron-replacement therapy and its long-term consequences on learning are not quite understood. A recent study in infants showed that the cognitive deficits in iron deficiency was reversed with iron supplementation. This finding provides evidence that in some settings cognitive deficits are not always permanent. Although further research is required to address these issues, several studies support the theory that iron sufficiency throughout the course of brain development is critical to normal brain iron and behavioural outcome.

\section{Introduction}

Iron deficiency continues to be the single most common nutritional deficiency and the main cause of anaemia in infancy and childhood. It is prevalent in most of the developing world and it is probably the only micronutrient deficiency of public health relevance in developed countries. The reported prevalence of iron-deficiency anaemia in young children varies in different populations, depending in part on the case definition used, with estimates ranging from $10 \%$ in Western societies to around $50 \%$ in less developed societies (1).

Numerous intervention studies have been performed across the world with varying success and it is clear that in nearly all situations it is a preventable disease with preventable consequences. One such consequence is the alteration in cognition that occurs in iron-deficient individuals during the early parts of their life cycle (2).

There is a history of more than 30 years of efforts to document the effects of iron deficiency on developmental delays in young children and infants (3-6).

Iron needs of the brain vary with the stage of the life cycle and the cell types in the central nervous system (CNS). Iron is the key component of the many enzymes that involve essential oxidation- reduction reactions, synthesis of neurotransmitters, catabolism of neurotransmitters and synthetic processes such as the production of myelin.

\section{Iron in the brain}

The highest levels of iron in the brain are found in the basal ganglia, but iron is found throughout the brain, including the white matter (7). Both biochemical and histochemical studies reveal white matter throughout the brain as a major site of iron concentration. Iron uptake into the brain is maximal during the period of rapid brain growth, which coincides with the peak of myelinogenesis. Lack of iron in the diet for the first 2 years of life has an important effect on development, as this is the time when the majority of brain growth occurs. Although the most rapid brain growth is seen in the months leading up to birth, at birth the brain has only reached $27 \%$ of its adult size and it continues to grow for the next 2 years (8). Iron levels in the brain at birth have been shown to be $10 \%$ of eventual adult levels, with the remainder accumulating through childhood and young adulthood (7). Recent research in humans points to impaired iron transport across the placenta in several prenatal conditions, e.g. diabetes mellitus, prenatal alcohol exposure, intrauterine growth retardation and ma- 
ternal stress. There is direct evidence of decreased brain iron or iron deficiency in the offspring (9). Infants born to mothers with nutritional iron deficiency in pregnancy are rarely anaemic, but they may have lower iron stores and develop iron efficiency sooner postnatally. There is now solid evidence that brain iron deficiency can occur even with a normal haemoglobin level.

Brain iron accumulates from birth to early adulthood (7). Perhaps for this reason, brain iron levels are more seriously affected by iron deficiency in the very young than in the adult animal. A brief period of severe iron deficiency in the young rat, but not in the adult, resulted in a deficit of brain iron that was not corrected by iron therapy, even though all signs of systemic iron deficiency were reversed (10). This resistance contrasts with the rapid normalization of hepatic iron and haemoglobin concentrations following iron repletion.

The failure to reverse iron depletion in the brain with iron treatment seems to be due to a slow rate of replacement of brain iron compounds (11).

\section{Possible explanatory mechanisms}

There has been considerable work on the possible mechanisms through which iron-deficiency anaemia affects cognitive function (12). Most emphasis has been placed on a direct neurochemical effect. Either iron deficiency causes low brain iron, which leads to a reduction in neurotransmitter levels, impaired transmitter function, hypomyelination and delayed neuromaturation, or the systemic effects of anaemia lead to low oxygen delivery to the brain, directly affect cognition. A conceptual model of these mechanisms is shown in Fig. 1. When iron deficiency occurs early in development in the rat, there are lasting deficits in brain iron, electrophysiological changes, a decrease in the number of dopamine $\mathrm{D}_{2}$ receptors and alterations in neurotransmitter function, hypomyelination and persisting behavioural changes that suggest an altered threshold of arousal (13).

The second hypothesis for the mechanism is that iron deficiency also has an indirect effect on behaviour. Iron-deficient anaemic infants and children have been shown to be less attentive and less responsive. Lower developmental scores may reflect poorer mother-child interaction because of the child's reduced responsiveness, and this could result in less effective stimulation of the environment. The lower scores may also reflect poorer interaction with the developmental assessor (14).

\section{Animal studies}

The development of iron-deficient animal models has been an invaluable tool for looking at the consequences of iron deficiency. Most animal studies have involved rats, as the distribution of iron in their brain is comparable to that of the human brain.

Weinberg et al. (15) were the first researchers to look at the behavioural consequences of iron deficiency. Iron-deficient rats had lower brain haem iron, were less active and responded less to environmental stimuli, compared with the control rats. Further testing revealed that iron-deficient rats showed an altered learning capacity (16). Learning capacity was also investigated by Yehuhda et al. (17) in postweanling rats made nutritionally iron deficient. The deficit in learning was established before any significant reduction in blood haemoglobin levels. After the iron-deficient rats were placed on a control diet for 3 weeks, there was a return to normal haemoglobin levels, but the learning capacity remained significantly reduced.

It must be recognized, however, that there are certain limitations in using animal models. Rats are less mature at birth, and humans and rats have different rates of neuronal development, both of which are particularly important when assessing the effect of early iron deprivation on mental function.

\section{Iron deficiency and neural functioning in humans}

Infancy is considered the age range of highest vulnerability for the CNS because it corresponds to the brain growth spurt and the unfolding of fundamental mental and motor processes.

Altered behaviour and development are among the most worrying concerns about iron deficiency in infancy, especially because this nutrient deficiency is most prevalent in the period between 6 and 24 months of age. Because this age range coincides with a period of maximal brain growth and the unfolding of many neurodevelopmental processes, several investigators have focused on the question of CNS effects of iron deficiency. The animal studies of brain iron content during development demonstrate that iron deficiency in early life (before weaning) is likely to have long-term effects that may be only partially reversible. In contrast, iron deficiency that 


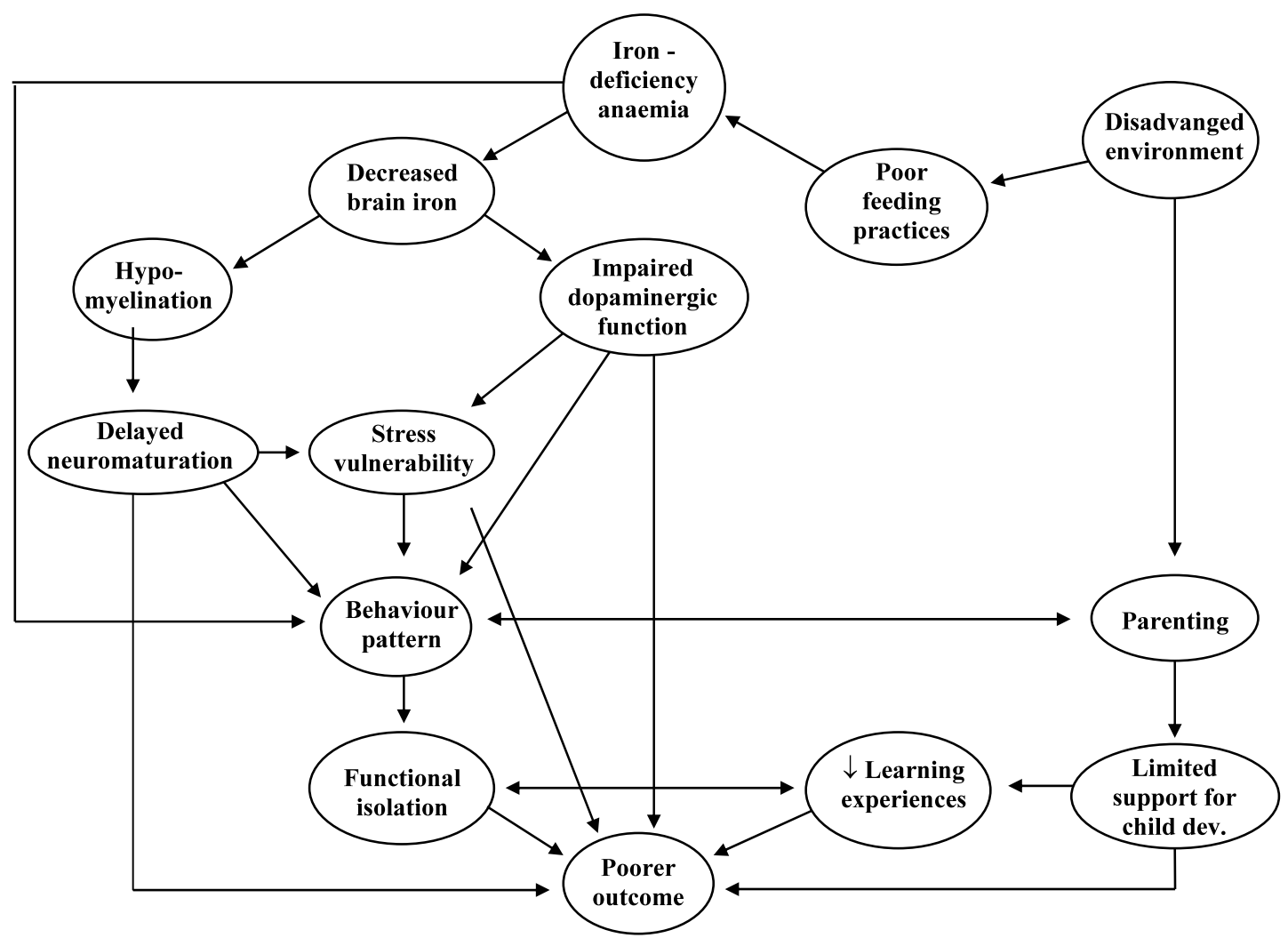

Fig. 1. Biological and environmental influences combine to produce poorer outcome (22).

occurs later in life (the postweaning model) also has severe effects, but they are readily reversible and of a different nature. Thus, the timing of iron deficiency seems critical to the severity and reversibility of its impact.

A recent review (18) identified 18 studies showing cross-sectional associations between iron-deficiency anaemia and poor cognitive function, motor development, behaviour or school achievement levels. However, the findings are totally not consistent and several studies have failed to find associations. Small samples and the confounding effect of protein-energy malnutrition may explain some of the negative findings.

\section{Longitudinal studies}

Longitudinal studies have found that children who were anaemic in the first 2 years of life continued to function poorly in later childhood. In Costa Rica (14), Chile (19) and Israel (20) children remained with poorer cognitive and motor function and school achievement levels. In general, the investigators controlled for many social background factors. Lozoff re-evaluated the children at 11-14 years of age (21). The children were free of iron deficiency and were growing normally by US standards. Children who had severe, chronic iron deficiency in infancy continue to have a behavioural and developmental disadvantage relative to peers more than 10 years after treatment.

Lozoff et al. investigated the possibility that irondeficient anaemic infants are "functionally isolated" (22). The behaviour of 52 Costa Rican 12-24month-old infants with iron-deficiency anaemia was contrasted with that of 139 control infants with better iron status. The investigators observed the infants during free play and also tested the infants with all the same motor and mental protocols. Infants with iron deficiency were more wary, hesitant and easily tired, made fewer attempts at test items, were less attentive to instructions and were less playful. In addition, the adults around the irondeficient infants interacted with them less. These data support the hypothesis that iron-deficient anaemic infants engage less with their environment. While this model also argues for the possibility of a widespread "nutritional stress" associated with decreased affect and activity, it does not exclude the possibility that direct biological effects of iron deficiency may result in these outcomes (Fig. 1). 
The evidence suggests that abnormally low levels of iron interfere with an organism's interaction with its environment, altering affective behaviour systems in sometimes subtle ways.

Studying the effects of single nutrient deficiencies on behaviour presents several problems. Deficiencies generally occur in conditions of relative poverty, and in developing countries they are often associated with protein-energy malnutrition and other micronutrient deficiencies, as well as infections.

\section{Nutrition and brain function}

Iron deficiency is caused by increased requirements for iron or reduced iron from the diet. Theoretically, therefore, some of the factors leading to an impaired iron balance may have an independent effect on brain function (Fig. 2). A further possibility is that a diet favouring the development of iron deficiency (a low content of available iron or an imbalance between dietary factors influencing iron absorption) may also be deficient in other nutrients (e.g. zinc) that are essential for adequate brain function. Such a diet may also contain an excess of some factors, which are deleterious for brain function. Examples are lead and cadmium, with an increased absorption caused by iron deficiency. In the analysis of the causal relationship between iron deficiency and brain function, several factors need to be considered. Hallberg (23) discussed several nutritional factors as possible confounders. The author concluded, however, that there is no strong support for the hypothesis that any of the nutritional factors identified are likely to account for the observations of a relationship between iron deficiency and brain function. However, more careful attention needs to be paid to the implications of the synergistic interactions between malnutrition and infection, and any change in the balance of factors determining iron absorption during an experiment may be a confounding factor.

\section{Supplementation}

The studies of Lozoff and those of Walter note a failure to improve performance in many of the anaemic children after iron therapy despite haematological normalization. The research team headed by Pollitt demonstrated a normalization of developmental scores in infants who were anaemic by providing a vigorous iron intervention for 4 months (24). While the reversibility of cognitive as opposed to developmental aspects of behaviour has yet to be demonstrated, this study provided encouraging evidence for the benefit of improving iron nutritional status in infants. According to recent research from Zanzibar, low-dose iron supplementation significantly improved the language and motor development of a community sample of rural African children (25). The impact of iron on language development did not seem to be related to any impact of anaemia. The results are encouraging because they suggest that the impact of iron deficiency on brain development can be partly reversed, even after the "window of vulnerability" for brain development between 9 and 24 months of age. The results are intriguing because they suggest that the relationship between iron and development

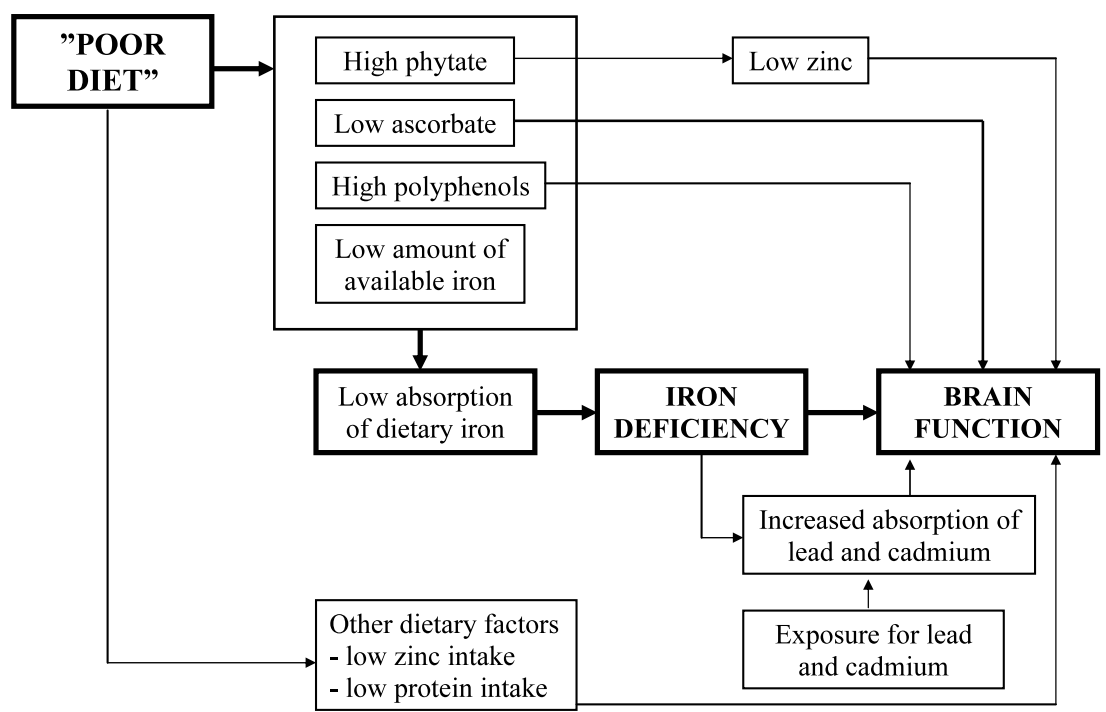

Fig. 2. Theoretical interrelationships between dietary factors and brain function (23). 
is subtle, and is different for linguistic skills than for psychomotor development. The former were susceptible to correction regardless of anaemia status, whereas the latter was only improved in the most severely anaemic children. There is also a need for the results to be confirmed in other studies.

\section{Conclusion}

A wealth of clinical, biochemical and neuropathological research shows that iron deficiency can exert a direct deleterious effect on learning and on the brain, and that this can occur with normal haemoglobin levels despite the confounding impact of socioeconomic factors. If subtle effects of iron deficiency in infancy lay the ground for later problems in cognitive and behavioural functioning, then a large, unrecognized population of children could be at risk owing to iron deficiency, a nutritional problem that needs to be prevented and treated.

\section{References}

1. De Maeyer EM, Adiels-Tegman M. The prevalence of anemia in the world. World Health Stat Q 1985; 38: 302-16.

2. Kretchner N, Beard JL, Carlson S. The role of nutrition in the development of normal cognition. Am J Clin Nutr 1995; 63: 997-1001S.

3. Beard JL, Connor JR, Jones BC. Iron in the brain. Nutr Rev 1993; 51: 57-71.

4. Beard JL, Connor JR, Jones BC. Brain iron: location and function. Prog Food Nutr Sci 1993; 17: 183-221.

5. Lozoff B. Behavioural alterations in iron deficiency. Adv Pediatr 1988; 35: 331-59.

6. Walter T. Impact of iron deficiency on cognition in infancy and childhood. Eur J Clin Nutr 1993; 47: 30716.

7. Hallgren B, Sourander P. The effect of age on the nonhaem iron in the human brain. J Neurochem 1958; 3: $41-51$.

8. Dobbing J, Sands J. Comparative aspects of the brain growth spurt. Early Hum Dev 1979; 3: 79-83.

9. Coe CL, Lutbach GR. Novel mechanism accounting for prenatal effects on the development of infant immunity. PNIRS Abstracts 1991; 2-12.

10. Dallman PR, Siimes M, Manies EC. Brain iron: persistent deficiency following short-term iron deprivation in the young rat. Br J Haematol 1975; 31: 209-15.

11. Dallman PR, Spirito RA. Brain iron in the rat: extremely slow turnover in normal rat may explain the long-lasting effects of early iron deficiency. J Nutr 1977; 107: $1075-81$.

12. Pollit E. Iron deficiency and cognitive function. Annu Rev Nutr 1993; 13: 521-37.

13. Felt BT, Lozoff B. Brain iron and behaviour of rats are not normalized by treatment of iron deficiency anemia during early development. J Nutr 1996; 126: 693-701.

14. Lozoff B, Jimenez E, Wolf AW. Long-term developmental outcome of infants with iron deficiency. N Engl J Med 1991; 325: 687-94.

15. Weinberg J, Dallman P, Levine S. Iron deficiency during early development in the rat: behavioural and physiological consequences. Pharmacol Biochem Beh 1980; 12: 493-502.

16. Weinberg J, Brett L, Levine S, Dallman P. Long-term effects of iron-deficiency on consummatory behaviour in the rat. Pharmacol Biochem Beh 1981; 14: 447-53.

17. Yehuda S, Youdim M, Mostofsky D. Brain irondeficiency causes reduced learning capacity in rats. Pharmacol Biochem Beh 1986; 25: 141-4.

18. Grantham-McGregor S, Ani C. A review of studies on the effect of iron deficiency on cognitive development in children. J Nutr 2001; 131: 649-8S.

19. De Andraca I, Walter T, Castillo $M$, et al. Iron deficiency anemia and its effect upon psychological development at preschool age: a longitudinal study. Nestlé Foundation Annual Report, 1990. Lausanne: Nestlé Foundation; 1990. p. 53-62.

20. Palti H, Meijer A, Adler B. Learning achievement and behaviour at school of anemic and non-anemic infants. Early Hum Dev 1985; 10: 217-23.

21. Lozoff B, Jimenez E, Hagen J, et al. Poorer behavioural and developmental outcome more than 10 years after treatment for iron deficiency in infancy. Pediatrics 2000; 105: $1-11$.

22. Lozoff B, Klein NK, Nelson EC, et al. Behaviour of infants with iron deficiency anemia. Child Dev 1998; 69: 24-36.

23. Hallberg L. Search for confounding factors in the relationship between iron deficiency and brain function. Am J Clin Nutr 1989; 50: 598-606.

24. Idjradinata P, Pollitt E. Reversal of developmental delays in iron-deficient anaemic infants treated with iron. Lancet 1993; 341: 1-4.

25. Stoltzfus RJ. Effects of iron supplementation and anthelmintic treatment on motor and language development of preschool children in Zanzibar. Double blind, placebo controlled study. Br Med J 2001; 323: 1389-93.

\footnotetext{
Lena Hulthén

Department of Clinical Nutrition

Sahlgrenska Academy at Göteborg University

SE-40530 Göteborg

Sweden

E-mail: lena.hulthen@medfak.gu.se
} 